\title{
INYECCIONES PERIURETRALES DE COPOLIMMERO DE DEXTRANÓMERO/ÁCIDO HIALURÓNICO COMO TRATAMIENTO DE LA INCONTINENCIA URINARIA DE ESFUERZO EN LA MUJER: DESCRIPCIÓN DE LA TÉCNICA Y REVISION DE LA LITERATURA.
}

\author{
J. Moreno Sierra, M. A. Alonso Prieto, L. Fernandez Montarroso, N. Pérez Romero, E. \\ Hernández Sánchez, I. Galante Romo, S. Prieto Nogal, J. Salinas Casado y A. Silmi Moyano.
}

Servicio de Urología. Hospital Clínico Universitario San Carlos. Universidad Complutense. Madrid. España.

Resumen.- OBJETIVO: El sistema permite un tratamiento no endoscopio y ambulatorio de la incontinencia urinaria de esfuerzo en la mujer. En este artículo se pretende conseguir la familiarización con el procedimiento.

METODOS: Descripción de la técnica de forma gráfica con los pasos correspondientes y revisión de la literatura.

RESULTADO: La inyección periuretral de copolimero de dextranomero/acido hialuronico como se deduce de la revisión de los principales estudios clínicos tiene una eficacia cercana al $75 \%$ con una técnica sencilla, reproducible y con escasos efectos adversos.

CONCLUSIONES: El tratamiento con este tipo de inyecciones periuretrales puede ser válido como manejo minimamente invasivo de la incontinencia urinaria de esfuerzo, por su eficacia, tolerabilidad y reproducibilidad.
Palabras clave: Incontinencia urinaria de esfuerzo. Inyección periuretral. Ácido hialuronico. Zuidex.

Summary.- OBJECTIVES: The system enables a non endoscopic, outpatient treatment of the female stress urinary incontinence. The article intends to familiarize the readers with the procedure.

METHODS: We describe the technique, graphically, with all steps, and perform a bibliographic review.

RESULTS: In accordance to the main clinical studies reviewed, the periurethral injection of dextranomer/ hyaluronic acid copolymer has an efficacy of near $75 \%$, with a simple, reproducible technique with rare adverse events.

CONCLUSIONS: The treatment with this type of periurethral injections may be valid as a minimally invasive treatment of the female stress urinary incontinence, because of its efficacy, tolerability and reproducibility.

Keywords: Stress urinary incontinence. Periurethral injection. Hyaluronic acid. Zuidex

\section{INTRODUCCIÓN}

Son muchas las técnicas que actualmente existen para el tratamiento de la incontinencia urinaria de esfuerzo femenina, pero a pesar de todo, también son muchas las mujeres con este problema que no buscan ayuda sanitaria para solucionarlo. $(1,2)$. Las principales razones que explican esta situación son la vergüenza y la percepción de la incontinen- 
cia como un problema menor o formando parte del proceso normal del envejecimiento. También existe incomodidad y reticencia a ser sometidas a una exploración uroginecologica $o$ incluso a ser intervenidas quirúrgicamente. (1).

Se ha publicado que la mayoría de las pacientes con incontinencia urinaria de esfuerzo en un sistema terciario de sanidad tenían predilección por procedimientos menos invasivos comparados con la cirugía invasiva si la probabilidad de curación con estos no estaba prácticamente garantizada. (3). En este sentido las inyecciones periuretrales como tratamiento minimamente invasivo de la incontinencia urinaria de esfuerzo pueden considerarse una opción válida, existiendo estudios que avala su eficacia y su seguridad. Las principales indicaciones para la utilización de está técnica son la incontinencia urinaria femenina de esfuerzo por hipermovilidad uretral, déficit intrinseco del esfínter y la incontinencia en mujeres con deseo genésico y fracaso o mala realización del tratamiento conservador.

En este artículo se describe la técnica de inyección periuretral de un copolímero de dextranómero/ácido hialurónico estabilizado de origen no animal (non-animal stabilized hyaluronic acid. NASHA).

\section{PROCEDIMIENTO}

\section{Material preciso}

El sistema consta de 4 jeringas llenas del gel de ácido hialurónico y un dispositivo de soporte, llamado IMPLACER que facilita su aplicación a través de la uretra. (Figura 1). Este es un dispositivo plástico estéril que consta de una pieza de mano con un cabezal y un tubo especialmente diseñado que tiene un soporte para la sujeción (Figura 2).

\section{Técnica paso a paso}

El procedimiento puede realizarse de forma ambulatoria o en Cirugía Mayor Ambulatoria (CMA), previa pauta de profilaxis antibiótica y explicación del procedimiento y obtención del consentimiento informado firmado. En él se debe explicar detalladamente que se va a proceder a implantar un agente periuretral biocompatible con la finalidad de intentar restaurar la función de la continencia y que puede requerir anestesia regional o local (más frecuente). Suele ser bien tolerado pero en ocasiones pueden ser necesarias nuevas aplicaciones o incluso precisar otras técnicas anti-incontinencia. Deberemos explicar a la paciente los posibles efectos adversos más frecuentes relacionados con la intervención (disuria, infección urinaria, dolor en el lugar de punción, sangrado, retención aguda de orina, desarrollo de granuloma, absceso y posible reacción de rechazo al material implantado).

\section{Primer paso: colocación y anestesia.}

Se coloca a la paciente en posición uroginecologica y se procede a la administración de anestesia local intrauretral con gel de Xilocaina.

\section{Segundo paso: ensamblaje del sistema.}

Mientras la anestesia hace efecto se procede al ensamblaje del sistema, introduciendo las cuatro jeringas en el dispositivo de soporte y aplicación llamado IMPLACER. (Figura 3).

\section{Tercer paso: medición de la longitud ure- tral.}

Antes de proceder a la inyección con este sistema, deberemos medir la longitud de la uretra con la finalidad de que el agente que se va a inyectar sea aplicado en la zona media uretral. Para realizar esto, introducimos una sonda Foley, llenando su balón que se situará a nivel del cuello vesical y marcaremos la porción de la sonda que aparece fuera de la uretra a nivel del meato. Esta medición nos servirá como guía, debiendo tener en cuenta que el IMPLACER debe ser introducido la mitad de lo medido, es decir colocado en uretra media.

\section{Cuarto paso: ubicación del implacer en ure- tra media.}

El siguiente paso de esta técnica consiste en introducir el IMPLACER, con el tubo inicialmente protegiendo las agujas (Figura 4, 5a, 5b), situandole a nivel de la uretra intermedia.

\section{Quinto paso: liberación de las agujas e in- yección de la sustancia en el interior de la uretra.}

Posteriormente se desplaza el tubo hacia atrás y de esta forma quedan liberadas las agujas en el interior de la uretra (Figura 6a, 6b). Posteriormente se procede a la introducción de las agujas en la mucosa uretral, inyectando los $0,7 \mathrm{ml}$ de copolímero que cada una de las agujas precargadas lleva en su interior (Figura 7), repitiéndose este proceso con el resto de las jeringas en sentido horario.

Una vez finalizado el proceso se deberá observar a la paciente durante treinta minutos y una vez 


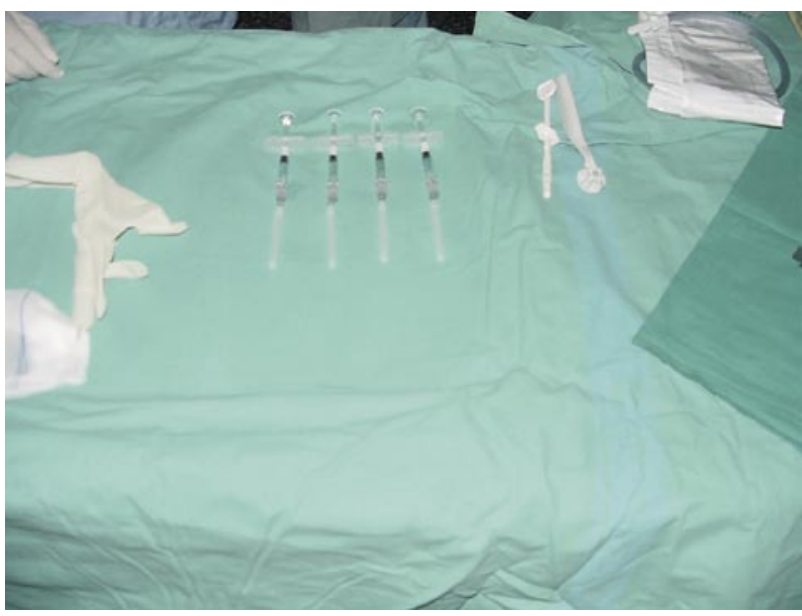

FIGURA 1. Jeringas precargadas y sistema IMPLACER.

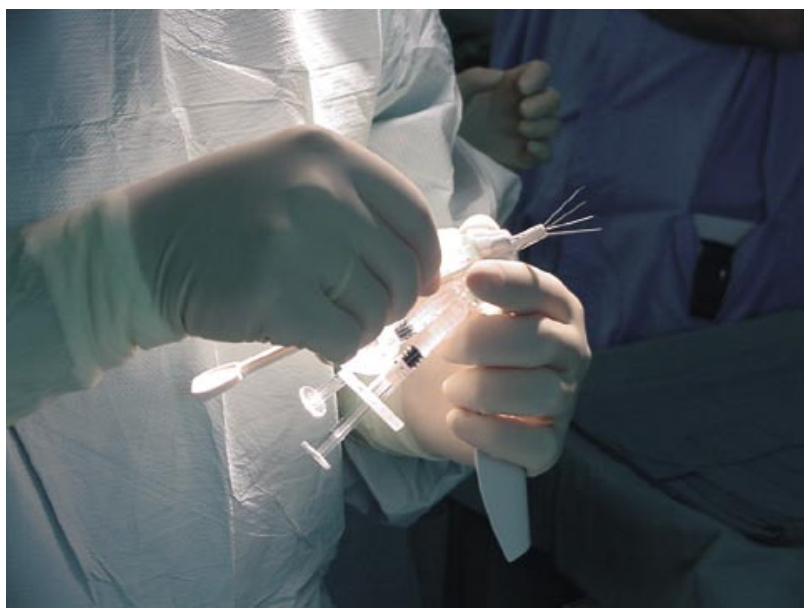

FIGURA 3. Disposición de las 4 jeringas en el sistema IMPLACER

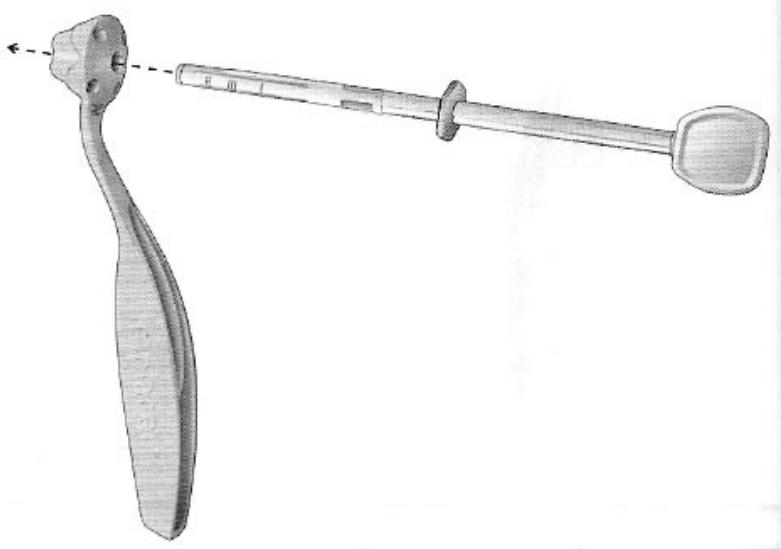

FIGURA 2. Colocación de las piezas del sistema IMPLACER.

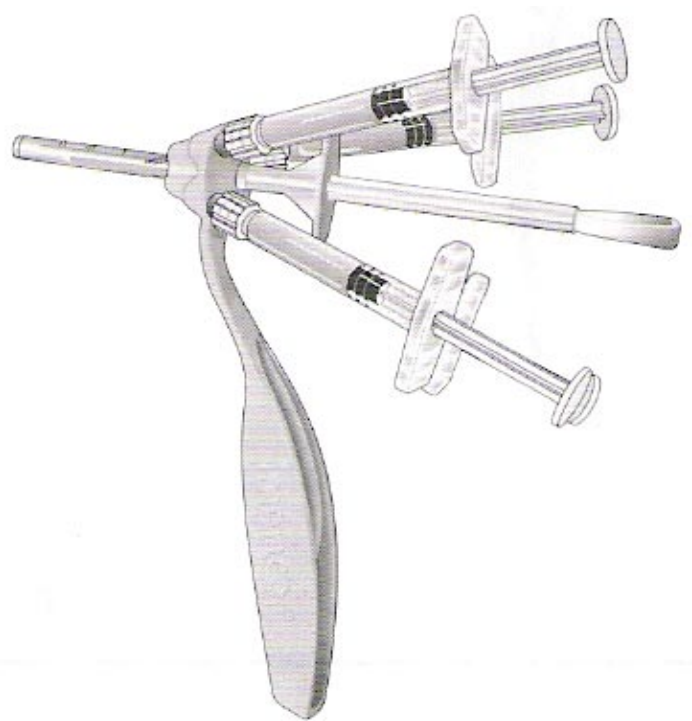

FIGURA 4. Sistema IMPLACER con agujas protegidas por un tubo.

material necesario para su estandarización sin usar en ningún momento control endoscópico, permitiendo de esta forma que este sea llevado a cabo de forma ambulatoria y no necesariamente en el quirófano.

Las inyecciones periuretrales con distintos agentes o materiales buscan aumentar la resistencia sobre la uretra y disminuir o eliminar los escapes de orina de forma involuntaria relacionados con el esfuerzo. En este sentido, hay evidencias clínicas que muestran que este tratamiento podría ser igualmente efectivo tanto en pacientes con hipermovilidad uretral como en la disfunción intrínseca del esfínter (5). Sea como fuere se necesitan estudios prospectivos que permitan demostrar su utilidad. A menudo tenemos 
pacientes con intención de quedarse embarazadas y tener más hijos, que presentan incontinencia urinaria de esfuerzo y no han respondido a un manejo conservador. En este caso defendemos la indicación de este procedimiento, ya que no hipoteca posteriores tratamientos. Además, recientemente se ha recomendado que el tratamiento conservador de la incontinencia urinaria de esfuerzo (entre el que se encuentra las inyecciones periuretrales), puede iniciarse sin necesidad de realizar un estudio urodinámico previo (6), lo que significa una simplificación del procedimiento.

El NASHA actúa principalmente como vehiculo de las microesferas de dextranómero hasta localizarlas en su lugar de implantación, de forma que van quedando envueltas gradualmente por el tejido conectivo de la paciente a quien se aplica, estimándose el tiempo de permanencia en unos 4 años aproximadamente.

En cuanto a la eficacia y tolerabilidad del copolímero NASHA, dos son los estudios clínicos desarrollados hasta el momento; el primero de ellos desarrollado por Van Kerrebroeck y cols (7), donde se objetiva una mejoría de los síntomas en la mayo-

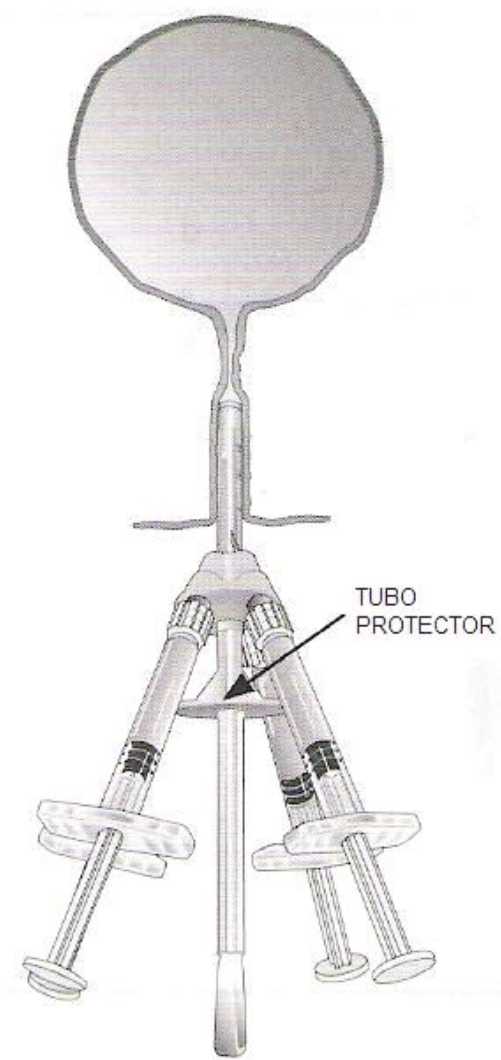

FIGURA 5A. Introducción del sistema en la uretra. ría de las pacientes $(76 \%)$ con buena tolerancia. Recientemente han sido publicados los resultados de un estudio multicéntrico, dirigido por Chapple, realizado sobre 142 pacientes (8), en el que se demuestra la utilidad de este procedimiento, con una eficacia objetiva del $77 \%$ a los 12 meses y una disminución superior o igual al $50 \%$ en un test de provocación de incontinencia, a la vez que observan una mejoría en la percepción subjetiva de la incontinencia por parte de las participantes. En aquellos casos en los que no se obtuvo un resultado satisfactorio inicial, se aplicó una nueva inyección periuretral 8 semanas después de la primera, ya que generalmente no se recomienda una segunda inyección antes de las 6 semanas.

Los principales efectos adversos que se han objetivado con este procedimiento son: retención aguda de orina (el más frecuente), infección del tracto urinario, urgencia urinaria, disuria, reacción y dolor en el sitio de inyección y pseudoquiste (Figura 8).

En principio la retención aguda de orina se soluciona con cateterismo vesical, y en la mayoría de las situaciones es autolimitada, siendo el resto de síntomas transitorios. En las infecciones del tracto urinario se administran antibióticos y en el caso de que aparezca un pseudoquiste este debe ser drenado y/ o resecado si ocasiona molestias a la paciente. (8)

Este procedimiento está indicado en todas aquellas pacientes en las que se ha comprobado incontinencia urinaria de esfuerzo mediante un test de provocación y hallan fallado otros tratamientos previos no invasivos, como pueden ser los ejercicios del suelo pélvico, biofeedback, estimulación eléctrica o

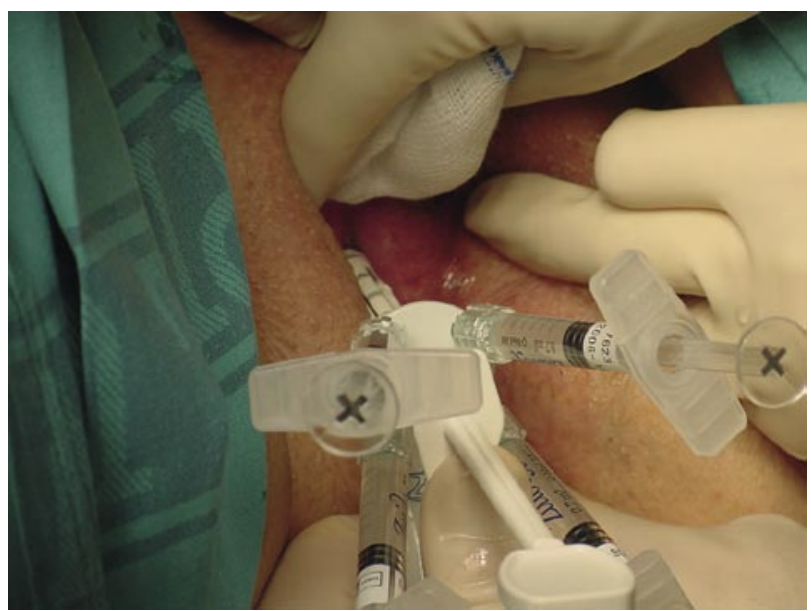

FIGURA 5B. Introducción real del sistema en la uretra. 


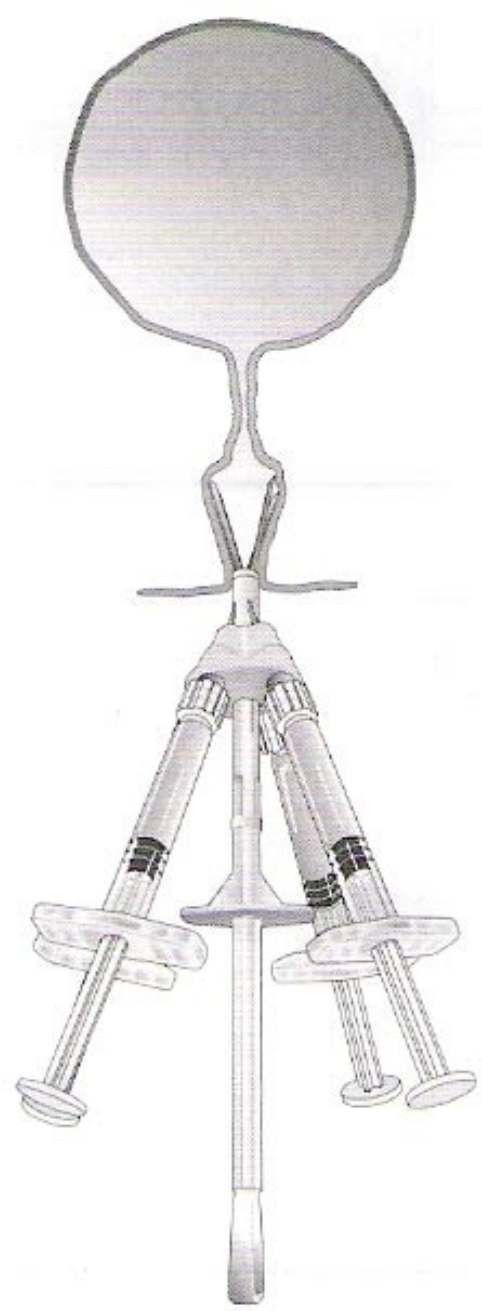

FIGURA 6A. Retirada del tubo protector del sistema.

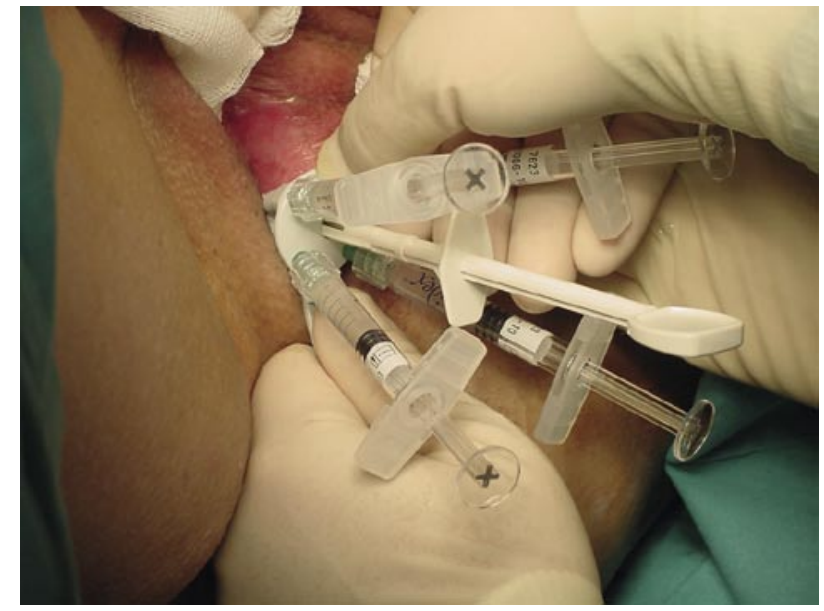

FIGURA 6B. Inserción de agujas en mucosa uretral.

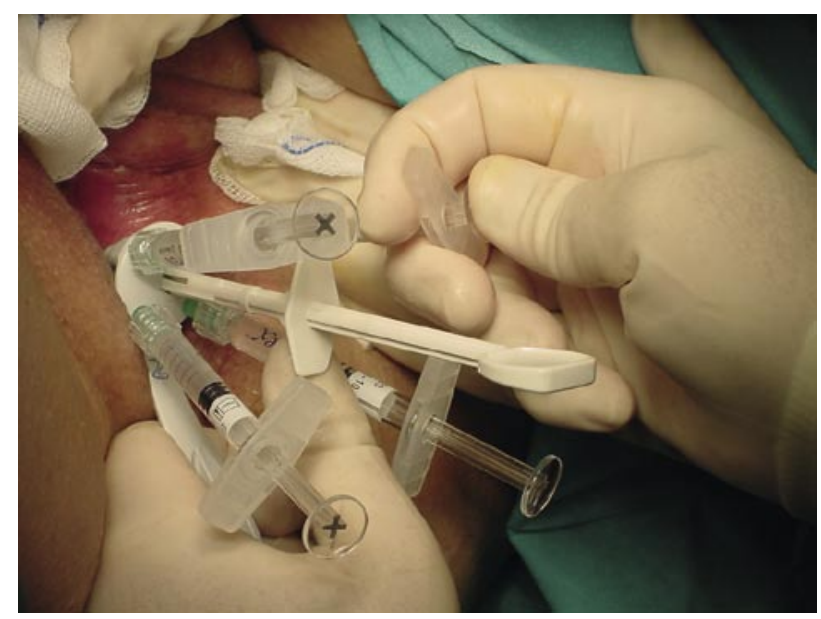

FIGURA 7. Inyección del copolímero.
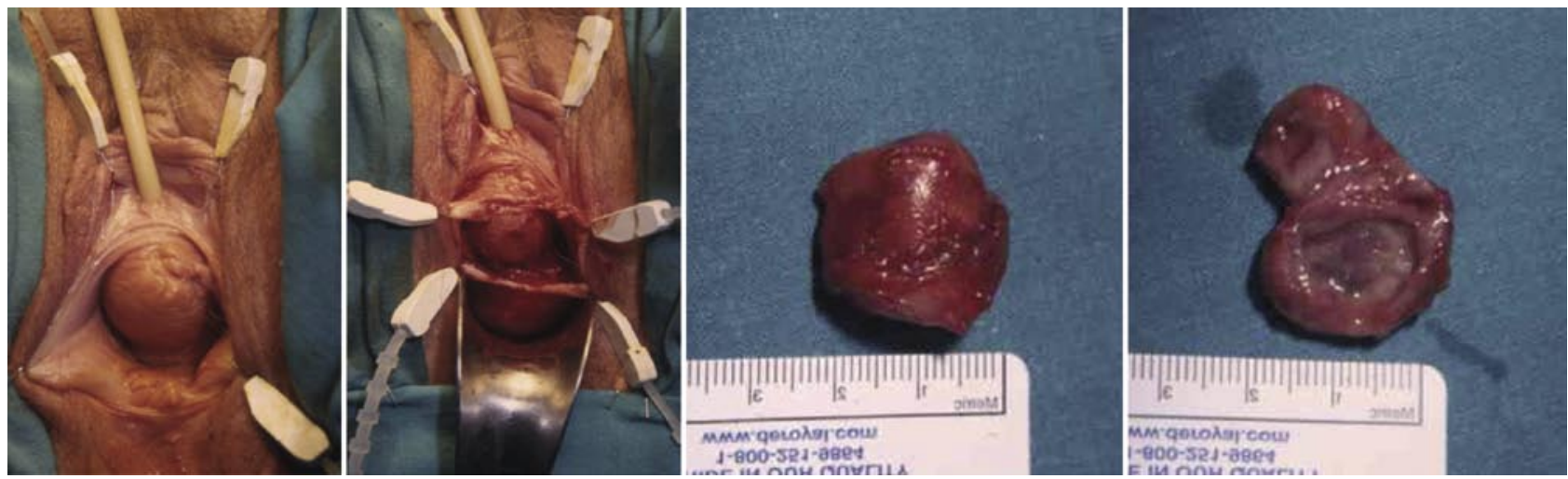

FIGURA 8. Pseudoquiste resecado.

tratamiento médico oral. No se recomienda cuado existe una afectación de la función vesical, esto es cuando existe hiperactividad vesical o lesiones neurológicas, cistocele, rectocele u otro tipo de prolapso o incluso cirugía del suelo pélvico previa. (8). El hecho de que exista infección del tracto urinario contraindica de forma absoluta su aplicación ya que estamos introduciendo un material exógeno en el organismo, que de esta forma puede infectarse complicando potencialmente de forma importante el procedimiento. 


\section{CONCLUSIONES}

1. La inyección periuretral con copolimero de dextranomero/acido hialuronico puede llevarse a cabo de forma sencilla y reproducible.

2. Se trata de un tratamiento minimamente invasivo, útil en la incontinencia urinaria de esfuerzo femenina, que puede ser aplicado de forma ambulatoria o incluso a nivel de una consulta de urología que disponga del equipamiento previso.

3. Los resultados en cuanto a la eficacia y la tolerabilidad son satisfactorios, aunque los escasos estudios realizados en cuanto a esta técnica, hacen necesario diseñar estudios prospectivos.

\section{BIBLIOGRAFÍA y LECTURAS RECOMENDADAS ( ${ }^{*}$ lectura de interés $y^{* *}$ lectura fundamental)}

1. ANDERSSON, G.; JOHANSSON, J.E.; GARPENHOLT, O. y cols.: "Urinary incontinenceprevalence, impact on daily living and desire for treatment: a population-based study". Scand. J. Urol. Nephrol.; 38: 125. 2004.

2. MARGALITH, I.; GILLON, G.; GORDON, D.: "Urinary incontinence in women under 65: quality of life, stress related to incontinence and patterns of seeking health care". Qual Life Res.; 13: 1381. 2004.

3. ROBINSON, D.; ANDERS, K., CARDOZO, L. y cols.: "What do women want? Interpretation of the concept of cure'. Pelvic Med Surg.; 9: 273. 2003.

4. LACKGREN, G.; WAHLIN, N.; SKOLDENBERG, E. y cols.: "Long-term followup of children treated whith dextranomer/hyaluronic acid copolymer for vesicoureteral reflux". J. Urol.; 166: 1887. 2001.

5. LIGHTNER, D.J., ITANO, N.B.; SWEAT, S.D. y cols.: "Injectable agents: present and future". Curr. Urol. Rep.; 3: 408. 2002.

**6. CHAPPLE, C.R.; WEIN, A.J.; ARTIBANI, W. y cols.: "A critical review of diagnosis criteria for evaluating patients with symptomatic stress urinary incontinence". BJU Int. 95: 327. 2005.

*7. VAN KERREBROECK, P.; TERMUELEN, F.; LARSSON, M.G. y cols.: "Efficacy and Safety of a novel system (NASHA/Dx copolymer using the Implacer device) for treatment of stress urinary incontinence". Urology.; 64: 276. 2004.

**8. CAPPLE, C.R.; HAAB, F.; CERVIGNI, M. y cols.: "An open, multicentre study of NASHA/Dx gel (Zuidex) for the treatment of stress urinary incontinence". Eur. Urol.; 48: 488. 2005. 